\title{
Small left atrial volume and dimension before ablation are predictors of tachycardia-induced cardiomyopathy with atrial fibrillation
}

\author{
Masahiro Nauchi ${ }^{1}$, Tsuyoshi Sakai ${ }^{1}$, Yuta Sugizaki ${ }^{1}$, and Naohiko Sahara ${ }^{1}$ \\ ${ }^{1}$ Saiseikai Yokohamashi Tobu Hospital
}

August 23, 2020

\begin{abstract}
Introduction Tachycardia-induced cardiomyopathy (TCM) is a reversible cause of heart failure with impaired left ventricle (LV) function. However, the diagnosis is difficult before treatment or control of the arrhythmia for the first time. This study was to clarify the characteristics of TCM with atrial fibrillation (AF) before AF ablation. Methods In this retrospective observational study, we observed 31 patients with paroxysmal or persistent AF who had impaired LV function without structural heart disease and who underwent catheter ablation. We defined impaired LV function as LV ejection fraction (LVEF) $<50 \%$ on the initial or worst echocardiogram. After ablation, the LVEF became $<60 \%$ (Group 1; $\mathrm{n}=9$ ) or [?]60\% (Group 2; $\mathrm{n}=22$ ). We compared the differences in baseline characteristics between the two groups. A receiver operating curve with area under the curve (AUC) was used to evaluate the prediction efficiency. The optimal cutoff point of the AUC was at which sensitivity and specificity would be maximal. Results There were significant differences in left atrial (LA) volume (LAV) by computed tomography (CT), LAV adjusted by body surface area (LAVI) by CT, LAVI by echocardiography, and LA diameter (LAD) (p < 0.05, respectively). The AUCs were 0.859, 0.869, 0.798, and 0.750, respectively. The optimal cutoff points were $147 \mathrm{ml}, 79 \mathrm{ml} / \mathrm{m} 2,37 \mathrm{ml} / \mathrm{m} 2$, and $45.8 \mathrm{~mm}$, respectively. Conclusion Small LAV, LAVI by CT, LAVI by echocardiography, and LAD were predictors of LVEF improvement. Small LA volume and dimension before ablation may be useful for diagnosis of TCM with AF.
\end{abstract}

Small left atrial volume and dimension before ablation are predictors of tachycardia-induced cardiomyopathy with atrial fibrillation

Masahiro Nauchi MD, Tsuyoshi Sakai MD, Yuta Sugizaki MD, Naohiko Sahara MD

Department of cardiovascular center, Saiseikai Yokohamashi Tobu Hospital

We express our thank to Akatsu, medical technologists, and members of Clinical research support center at Saiseikai Yokohamashi Tobu Hospital. We would like to thank Enago (www.enago.jp) for the English language review.

The authors declare no conflicts of interest associated with this manuscript.

Corresponding Author: Masahiro Nauchi

Saiseikai Yokohamashi Tobu Hospital

3-6-1 Shimosueyoshi, Tsurumi Ward, Yokohama city, Kanagawa, Japan.

E-mail address:m_nauchi@tobu.saiseikai.or.jp

Tel +81455763000

Fax +81455763525 


\section{Abstract}

Introduction

Tachycardia-induced cardiomyopathy (TCM) is a reversible cause of heart failure with impaired left ventricle (LV) function. However, the diagnosis is difficult before treatment or control of the arrhythmia for the first time. This study was to clarify the characteristics of TCM with atrial fibrillation (AF) before AF ablation.

Methods

In this retrospective observational study, we observed 31 patients with paroxysmal or persistent AF who had impaired LV function without structural heart disease and who underwent catheter ablation. We defined impaired LV function as LV ejection fraction (LVEF) $<50 \%$ on the initial or worst echocardiogram. After ablation, the LVEF became $<60 \%$ (Group 1; $\mathrm{n}=9$ ) or [?]60\% (Group 2; $\mathrm{n}=22$ ). We compared the differences in baseline characteristics between the two groups. A receiver operating curve with area under the curve (AUC) was used to evaluate the prediction efficiency. The optimal cutoff point of the AUC was at which sensitivity and specificity would be maximal.

Results

There were significant differences in left atrial (LA) volume (LAV) by computed tomography (CT), LAV adjusted by body surface area (LAVI) by CT, LAVI by echocardiography, and LA diameter (LAD) (p < 0.05 , respectively). The AUCs were $0.859,0.869,0.798$, and 0.750 , respectively. The optimal cutoff points were $147 \mathrm{ml}, 79 \mathrm{ml} / \mathrm{m}^{2}, 37 \mathrm{ml} / \mathrm{m}^{2}$, and $45.8 \mathrm{~mm}$, respectively.

Conclusion

Small LAV, LAVI by CT, LAVI by echocardiography, and LAD were predictors of LVEF improvement. Small LA volume and dimension before ablation may be useful for diagnosis of TCM with AF.

Key words: Tachycardia-induced cardiomyopathy, Arrhythmia-induced cardiomyopathy, atrial fibrillation, catheter ablation, heart failure

Abbreviations

TCM, tachycardia induced cardiomyopathy

$\mathrm{HF}$, heart failure

$\mathrm{LV}$, left ventricular/ventricle

$\mathrm{AF}$, atrial fibrillation

$\mathrm{EF}$, ejection fraction

DCM, dilated cardiomyopathy

CMR, cardiac magnetic resonance

CT, computed tomography

LA, left atrial/atrium

LAV, left atrial volume

LAVI, left atrial volume index

BSA, body surface area

$\mathrm{PV}$, pulmonary vein

LAA, left atrial appendage

PVI, pulmonary vein isolation 
CIED, cardiac implantable electronic device

ROC, receiver operating characteristic

AUC, area under curve

CI, confidence interval

LAD, left atrial diameter

1 Introduction

TCM is one of Arrhythmia-induced cardiomyopathies and a reversible cause of HF with impaired LV function. $\mathrm{AF}$ is the most common cause of $\mathrm{TCM}^{1,2}$. We cannot always observe echocardiographic data in typical TCM at the first occurrence: LVEF is $<30 \%, \mathrm{LV}$ end-diastolic diameter is $<65 \mathrm{~mm}$, and LV end-systolic diameter is $<50 \mathrm{~mm}^{3}$. The diagnosis is determined by a recovery of LV function within 3 months after treatment or control of the arrhythmia ${ }^{4}$. In other words, we cannot determine the diagnosis for at least 3 months after arrhythmia control. Moreover, the diagnosis is determined by excluding other causes of cardiomyopathy, especially idiopathic DCM. Absence of late Gadolinium enhancement on CMR imaging has been useful for excluding other causes ${ }^{5}$. However, it is an unusual method and is difficult for patients with impaired renal function.

Thus, the purpose of this study was to clarify the characteristics of TCM with AF before AF ablation. These should help establish early and optimal examinations, such as transesophageal echocardiography or CT, and treatments, such as AF ablation or cardioversion.

2 Methods

2.1 Study design and study population

Hosted file

image1.emf available at https://authorea.com/users/348483/articles/476674-small-leftatrial-volume-and-dimension-before-ablation-are-predictors-of-tachycardia-inducedcardiomyopathy-with-atrial-fibrillation

This was a single-center, retrospective analysis of patients with AF who had impaired LV function (LVEF $<50 \%$ ) on the initial or worst echocardiogram before ablation and who underwent catheter ablation for the AF at Saiseikai Yokohamashi Tobu Hospital from June 2016 to January 2020. We excluded patients receiving hemodialysis or who had structural heart disease and suspicion of other cardiomyopathies by echocardiography. After control of the AF, the patients were divided into two groups based on whether the LVEF was $<60 \%$ (Group 1) or [?]60\% (Group 2) more than 3 months after ablation. If the patient's LVEF became [?]60\% within 3 months after ablation, the patient was included in Group 2. We compared the baseline characteristics between the two groups. This study was conducted at Saiseikai Yokohamashi Tobu Hospital and was approved by the institutional review board of Saiseikai Yokohamashi Tobu Hospital (IRB \# 20200050).

\subsection{Echocardiographic data}

Echocardiography was performed using various devices (Vivid E95, GE Healthcare Japan Corp.; APLIO Artida, APLIO I900, and APLIO 300, Canon Medical Systems Corp.; and iE, Philips Japan, Ltd.). LVEF and LAV were measured by the modified Simpson's method and by the prolate-ellipsoid method (LAV-echo), respectively. Division of the LAV-echo by the BSA resulted in LAV index of echocardiography (LAVI-echo). If LVEF became [?]60\% within 3 months after ablation, the data were adopted. If the patient had a recurrence after ablation, echocardiography was performed over 3 months after the arrhythmia resolved.

2.3 CT data 
We performed CT before ablation. We utilized the CT data, and the anatomy of LA and PVs was constructed by Ziostation2 Version 2.9.7.1 (Ziosoft, Inc). Vertical and horizontal rotation was performed to maximize the length between the PV roof and the first bifurcation of PV. The LA-PV junction was determined as previously reported ${ }^{6,7,8}$. The LA-LAA junction was defined as the root of LAA. LAV determined by CT (LAV-CT) was removed of the LAA and PVs by a manual procedure at these junctions (Figure 1). Division of the LAV by the BSA resulted in the LAV index of CT (LAVI-CT).

\subsection{Ablation procedure}

Patients received intravenous heparin with a target minimum activated clotting time of 300 seconds. AF ablation was performed using a cryoballoon (Arctic Front Advance; Medtronic, Inc) for PVI and a 3-dimentional electroanatomic mapping system (EnSite Precision; Abbott, Inc) in all patients. If the PVI was incomplete, complete PVI was performed with an irrigated radiofrequency catheter (FlexAbility; Abbott, Inc). Cavotricuspid isthmus line ablation was completed with the radiofrequency catheter.

\subsection{Post-ablation follow-up}

A 12-lead electrocardiogram was performed at every visit. 24-hour Holter monitoring and echocardiography were performed according to the physician's judgment. If patients had a CIED, AF was monitored. Biopsy and CMR imaging were not performed. Recurrence of AF is defined as longer than 30 seconds of $\mathrm{AF}$ or atrial tachycardia/flutter episode more than 3 months after ablation.

\subsection{Statistical analysis}

Continuous data were described as median and interquartile range and categorical data as numbers and percentages. The Mann-Whitney $\mathrm{U}$ test and Fisher exact test were used to compare differences across groups. All tests were 2 sided, and a p value $<0.05$ was considered to indicate statistical significance. A ROC curve was calculated to identify the optimal cutoff point where sensitivity and specificity would be maximal for the prediction of improved LVEF. The AUC and its 95\% CI were calculated as measures of the accuracy of the data. Statistical analysis was performed using IBM SPSS Advanced Statistics 24 (IBM, Inc). We could not perform multivariable analysis because of the small amount of data.

3 Results

\subsection{Characteristics of 2 groups at baseline and predictors for improving LVEF}

A total of 31 patients (nine in Group1 and 22 in Group 2) were included in this study. One patient in Group 1 and eight patients in Group 2 had AF controlled by cardioversion and/or medications before ablation. In Group 1, one patient had a CIED before ablation and two patients had it after ablation. In Group 2, nobody had CIED. LAV-CT, LAVI-CT, LAVI-echo, and LAD were significant between the groups. Small LAV-CT, LAVI-CT, LAV-echo, and LAD were predictors for improving LVEF. All other characteristics were not significantly different between the groups (Table 1). The AUCs of LAV-CT, LAVI-CT, LVAI-echo, and LAD were 0.859 (95\% CI 0.729-0.991, $\mathrm{p}=0.002), 0.869$ (95\% CI 0.741-0.997, $\mathrm{p}=0.001), 0.798$ (95\% CI 0.640$0.956, \mathrm{p}=0.010$ ), and 0.750 (95\% CI $0.545-0.955, \mathrm{p}=0.031$ ), respectively. The cutoff values were $147 \mathrm{ml}, 79$ $\mathrm{ml} / \mathrm{m}^{2}, 37 \mathrm{ml} / \mathrm{mm}^{2}$, and $45.8 \mathrm{~mm}$, respectively (Figure 2 ).

\subsection{Characteristics of 2 groups after ablation}

Post-ablation echocardiographic data were not significantly different between the groups except for the follow up period of echocardiography and LAD. Recurrence of arrhythmia was not significantly different between the groups (Table 2).

4 Discussion

\subsection{Major finding}

Several pieces of previous literature reported that small LV dimensions, younger age, and male sex may help to differentiate TCM from $\mathrm{DCM}^{3,4,9,10}$. These parameters in this study were not significantly different 
between the two groups. LAV-CT, LAVI-CT, LAVI-echo, and LAD were significantly different, whereas the difference in the LAV-echo was close to significant $(\mathrm{p}=0.0583)$. LA dilation is frequently observed in DCM because of diastolic dysfunction, functional mitral regurgitation, AF, and LV cavity enlargement ${ }^{11}$. Therefore, small LA volume and dimension may be predictors of TCM with AF. Small LAV-echo and LAD (LAV-echo $<67 \mathrm{ml}$ and $\mathrm{LAD}<45.8 \mathrm{~mm}$, which were the optimal cutoff values) may be helpful for the diagnosis of TCM with AF, because echocardiography is noninvasive and easy. However, these results of this study may not apply to TCM with other arrhythmias.

\subsection{AF ablation}

$\mathrm{AF}$ is the most prevalent arrhythmia and the most common cause of $\mathrm{TCM}^{1,2}$, but there is no clear data on the prevalence of $\mathrm{TCM}^{12}$. TCM in $4 \%$ of patients referred for PVI was reported ${ }^{13}$. Ventricular rate during AF does not predict for LVEF improvement ${ }^{14}$. Thus, it is difficult the diagnosis of TCM with AF. In case of other supraventricular arrhythmias, we can recognize that they cause TCM and we can recommend ablation $^{3,15,16,17}$. All patients with $\mathrm{HF}$ and $\mathrm{AF}$ should have ablation for $\mathrm{AF}^{18,19}$, but it is prevented by the risk of the procedure's complications ${ }^{20,21,22,23}$ : Phrenic nerve injury, PV stenosis, Atrioesophageal fistula, and so on. Hence, we recognize some characteristics of TCM before ablation, and we are willing to recommend patients with possible TCM for ablation.

\subsection{Variety in Group 1}

Patients in Group 1 were classified into two groups. In Group A, their LVEF improved a little. In group B, their LVEF remained the same or decreased. Group A was composed of the following patients: (1) those whose LVEF had been improving, (2) those with overlapping DCM and $\mathrm{TCM}^{1}$, and (3) those with recurrence of AF. Group B was composed of just DCM or other cardiomyopathies. Accordingly, we should have followed Group 1 longer.

\subsection{Limitations}

This study has several limitations. It was a single-center study and included a small number of patients. LAV-CT was measured by hand and it might not be exact. Some tests, such as echocardiography, were performed according to individual judgment. Although three patients (33\%) in Group 1 had CIED in the end, we did not notice the occult recurrence of AF.

5 Conclusion

LAV-CT $<147 \mathrm{ml}$, LAVI-CT $<79 \mathrm{ml} / \mathrm{m}^{2}$, LAVI- echo $<37 \mathrm{ml} / \mathrm{m}^{2}$, and LAD $<45.8 \mathrm{~mm}$ were predictors of LVEF improvement. The parameters measured by CT were more useful than by echocardiography. Small LAV and dimension before ablation may be predictors for TCM with AF and may be helpful for its diagnosis.

Conflict of interests

The authors declare that there are no conflicts of interests.

References

1. Gopinathannair R, Etheridge SP, Marchlinski FE, Spinale FG, Lakkireddy D, Olshansky B. Arrhythmia-induced cardiomyopathy: Mechanisms, recognition and management. J Am Coll Cardiol 2015;66:1714-1728.

2. Nerheim P, Birger-Botkin S, Piracha L, Olshansky B. Heart failure and sudden death in patients with tachycardia-induced cardiomyopathy and recurrent tachycardia. Circulation 2004;110:247-252.

3. Medi C, Kalman JM, Haqqani H, Vohra JK, Morton JB, Sparks PB, Kistler PM. Tachycardia-mediated cardiomyopathy secondary to focal atrial tachycardia: long-term outcome after catheter ablation. J Am Coll Cardiol 2009;53:1791-1797.

4. Lishmanov A, Chockalingam P, Senthilkumar A, Chockalingam A. Tachycardia-induced cardiomyopathy: evaluation and therapeutic options. Congest Heart Fail 2010;16:122-126. 
5. Hasdemir C, Yuksel A, Camli D, Kartal Y, Simsek E, Musayev O, Isayev E, Aydin M, Can LH. Late gadolinium enhancement CMR in patients with tachycardia-induced cardiomyopathy caused by idiopathic ventricular arrhythmia. Pacing Clin Electrophysiol 2012;35:465-470.

6. Shah DC, Haïssaguerre M, Jaïs P, Hocini M, Yamane T, Deisenhofer I, Garrigue S, Clémenty J. Curative catheter ablation of paroxysmal atrial fibrillation in 200 patients: strategy for presentations ranging from sustained atrial fibrillation to no arrhythmias. Pacing Clin Electrophysiol 2001; 4:1541-1558.

7. Shah DC.Evaluating pulmonary vein isolation. J Arrhythmia 2008;24:180-194.

8. Manghat NE, Mathias HC, Kakani N, Hamilton MC, Morgan-Hughes G, Roobottom CA. Pulmonary venous evaluation using electrocardiogram-gated 64-detector row cardiac CT. Br J Radiol 2012;85:965971.

9. Jeong YH, Choi KJ, Song JM, Hwang ES, Park KM, Nam GB, Kim JJ, Kim YH. Diagnostic approach and treatment strategy in tachycardia-induced cardiomyopathy. Clin Cardiol 2008;31:172-178.

10. Ju W, Yang B, Li M, Zhang F, Chen H, Gu K, Yu J, Cao K, Chen M. Tachycardiomyopathy complicated by focal atrial tachycardia: incidence, risk factors, and long-term outcome. J Cardiovasc Electrophysiol 2014;25:953-957.

11. Japp AG, Gulati A, Cook SA, Cowie MR, Prasad SK. The diagnosis and evaluation of dilated cardiomyopathy. J Am Coll Cardiol 2016;67:2996-3010.

12. Huizar JF, Ellenbogen AK, Tan AY, Kaszala K. Arrhythmia-induced cardiomyopathy: JACC state-ofthe-art review. J Am Coll Cardiol 2019;73:2328-2344.

13. Gentlesk PJ, Sauer WH, Gerstenfeld EP, Lin D, Dixit S, Zado E, Callans D, Marchlinski FE. Reversal of left ventricular dysfunction following ablation of atrial fibrillation. J Cardiovasc Electrophysiol 2007;18:9-14.

14. Redfield MM, Kay GN, Jenkins LS, Mianulli M, Jensen DN, Ellenbogen KA. Tachycardia-related cardiomyopathy: a common cause of ventricular dysfunction in patients with atrial fibrillation referred for atrioventricular ablation. Mayo Clin Proc 2000;75:790-795.

15. Luchsinger JA, Steinberg JS. Resolution of cardiomyopathy after ablation of atrial flutter. J Am Coll Cardiol 1998;32:205-210.

16. Han FT, Riles EM, Badhwar N, Scheinman MM. Clinical features and sites of ablation for patients with incessant supraventricular tachycardia from concealed nodofascicular and nodoventricular tachycardias. JACC Clin Electrophysiol 2017;3:1547-1556.

17. Bensler JM, Frank CM, Razavi M, Rasekh A, Saeed M, Haas PC, Nazeri A, Massumi A. Tachycardiamediated cardiomyopathy and the permanent form of junctional reciprocating tachycardia. Tex Heart Inst J 2010;37:695-698.

18. Marrouche NF, Brachmann J, Andresen D, Siebels J, Boersma L, Jordaens L, Merkely B, Pokushalov E, Sanders P, Proff J, Schunkert H, Christ H, Vogt J, Bänsch D. Catheter ablation for atrial fibrillation with heart Failure. N Engl J Med 2018;378:417-427.

19. Prabhu S, Taylor AJ, B.T. Costello BT, Kaye DM, McLeiian AJA, Voskoboinik A, Sugumar H, Lockwood SM, Stokes MB, Pathik B, Nalliah CJ, Wong QR, Azzopardi SM, Gutman SJ, Lee G, Layland J, Mariani JA, Ling LH, Kalman JM, Kistler PM. Catheter Ablation Versus Medical Rate Control in Atrial Fibrillation and Systolic Dysfunction: The CAMERA-MRI Study. J Am Coll Cardiol 2017;70:19491961.

20. Kuck KH, Brugada J, Furnkranz A, Metzer A, Ouyang F, Chun KRJ, Elvan A, Arentz T, Bestehorn K, Pocock SJ, Albenque JP, Tondo C, FIRE AND ICE Investigators. Cryoballoon or radiofrequency ablation for paroxysmal atrial fibrillation. N Engl J Med 2016;374: 2235-2245.

21. Okumura K, Matsumoto K, Kobayashi Y, Nogami A, Hokanson RB, Kueffer F, CRYO-Japan PMS Study Investigators. Safety and efficacy of cryoballoon ablation for paroxysmal atrial fibrillation in Japan - Results from the Japanese prospective post-market surveillance study. Circ J 2016;80:17441749 .

22. Narui R, Tokuda M, Matsushima M, Isogai R, Tokutake K, Yokoyama K, Hioki M, Ito K, Tanigawa S, Yamashita S, Inada K, Shibayama K, Matsuo S, Miyanaga S, Sugimoto K, Yoshimura M, Yamane $\mathrm{T}$. Incidence and factors associated with the occurrence of pulmonary vein narrowing after cryoballoon 
ablation. Circ Arrhythm Electrophysiol 2017;10:e004588.

23. Kawasaki R, Gauri A, Elmouchi D, Duggal M, Bhan A. Atrioesophageal fistula complicating cryoballoon pulmonary vein isolation for paroxysmal atrial fibrillation. J Cardiovasc Electrophysiol 2014;25:787-792.

(a)

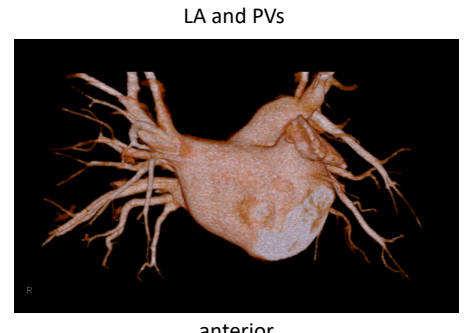

anterior

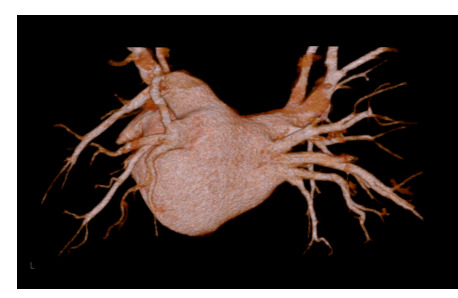

posterior (b)
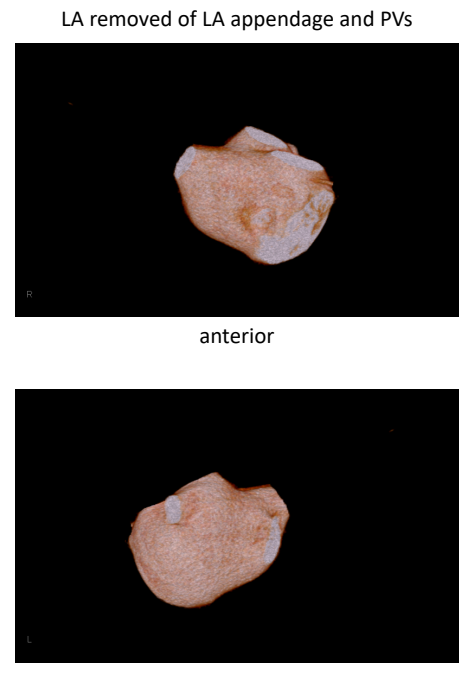

posterior 
Table 1. Characteristics of 2 groups at baseline

\begin{tabular}{|c|c|c|c|}
\hline & Group 1 & Group 2 & $p$ value \\
\hline & $\mathrm{n}=9$ & $\mathrm{n}=22$ & \\
\hline Age - yr & $68(60-72)$ & $60(50-67)$ & 0.064 \\
\hline Male & $7(77.8)$ & $16(72.7)$ & 1 \\
\hline Body mass index $-\mathrm{kg} / \mathrm{m}^{2}$ & $25.1(22.6-26.5)$ & $24.4(22.3-27.8)$ & 1 \\
\hline Body surface area - $\mathrm{m}^{2}$ & $1.66(1.55-1.77)$ & $1.81(1.71-1.90)$ & 0.0778 \\
\hline \multicolumn{4}{|l|}{ New York Heart Association class } \\
\hline I & $4(36.4)$ & $8(38.1)$ & \\
\hline II & $5(45.5)$ & $5(23.8)$ & \\
\hline III & $2(18.2)$ & $8(38.1)$ & \\
\hline IV & $0(0)$ & $0(0)$ & \\
\hline \multicolumn{4}{|l|}{ Type of AF } \\
\hline Paroxysmal & $1(11.1)$ & $0(0)$ & \\
\hline Persistent & $6(66.6)$ & $19(86.4)$ & \\
\hline Long standing persistent & $2(22.2)$ & $3(13.6)$ & \\
\hline CHADS2 & $2(2-3)$ & $1.3(1.3-2.8)$ & 0.78 \\
\hline CHA2DS2-VASC & $3(2-4)$ & $2.5(2-3)$ & 0.489 \\
\hline \multicolumn{4}{|l|}{ echocardiographic parameters } \\
\hline Heart rate during AF - bpm & $91(74-108)$ & $97(84-116)$ & 0.317 \\
\hline LAD - mm & $49.2(43.1-52.5)$ & $44.5(38.4-45.6)$ & 0.0329 \\
\hline LAV - $\mathrm{ml}$ & $76.5(71.4-95.5)$ & $69.2(54.8-79.9)$ & 0.0583 \\
\hline LAVI $-\mathrm{ml} / \mathrm{m}^{2}$ & $46.1(42.0-57.5)$ & $33.3(29.8-43.4)$ & 0.00899 \\
\hline LVEF - \% & $34.0(23.0-43.0)$ & $37.3(27.5-42.9)$ & 0.632 \\
\hline LV end-diastolic diameter - $\mathrm{mm}$ & $53.2(53.2-61.9)$ & $50.7(47.2-53.4)$ & 0.286 \\
\hline LV end-systolic diameter - mm & $45.7(34.9-48.1)$ & $42.9(34.9-48.1)$ & 0.157 \\
\hline Moderate or severe MR & $4(44.4)$ & $4(18.2)$ & 0.185 \\
\hline \multicolumn{4}{|c|}{ computed tomographic parameters } \\
\hline LAV - ml & 165(148-186) & 141(118-157) & 0.00214 \\
\hline LAVI $-\mathrm{ml} / \mathrm{m}^{2}$ & $101(90-125)$ & $76(64-88)$ & 0.000834 \\
\hline Time from $\mathrm{CT}$ to ablation - $\mathrm{d}$ & $10(5-11)$ & $13(10-15)$ & 0.0958 \\
\hline NT-proBNP - pg/ml & $719(685-2096)$ & $807(456-1304)$ & 0.623 \\
\hline $\mathrm{Hb}-\mathrm{g} / \mathrm{dl}$ & $14.0(13.5-14.6)$ & $14.6(14.1-16.0)$ & 0.138 \\
\hline eGFR $-\mathrm{ml} / \mathrm{min} / 1.73 \mathrm{~m}^{2}$ & $59(54-65)$ & $65(56-76)$ & 0.42 \\
\hline Hypertension & $6(66.7)$ & $16(72.7)$ & 1 \\
\hline Diabetes & $2(22.2)$ & $4(18.2)$ & 1 \\
\hline Chronic kidney disease & $1(11.1)$ & $3(13.6)$ & 1 \\
\hline Dyslipidemia & $4(44.4)$ & $8(36.4)$ & 0.704 \\
\hline Sleep apnea syndrome & $1(11.1)$ & $2(9.1)$ & 1 \\
\hline Alcohol consumption & $5(55.6)$ & $14(63.6)$ & 0.704 \\
\hline Current smoker & $0(0)$ & $6(27.3)$ & 0.145 \\
\hline Beta-blocker use & $8(88.9)$ & $21(95.5)$ & 0.503 \\
\hline ARB or ACE inhibitor use & $6(66.7)$ & $9(40.1)$ & 0.252 \\
\hline MRA use & $7(77.8)$ & 13(59.1) & 0.429 \\
\hline Loup diuretic use & $8(88.9)$ & 13(59.1) & 0.205 \\
\hline Amiodarone use & $2(22.2)$ & $5(22.7)$ & 1 \\
\hline Bepridil use & $2(22.2)$ & $5(22.7)$ & 1 \\
\hline Digitalis use & $0(0)$ & $1(4.5)$ & 1 \\
\hline CIED & $1(11.1)$ & $0(0)$ & 0.29 \\
\hline
\end{tabular}

Abbreviations: $A F$, atrial fibrillation; $L A D$, left atrial diameter; $L A V$, left atrial volume; LAVI, left atrial volume index; LVEF, left ventricular ejection fraction; LV, left ventricular; MR, mitral regurgitation; $\mathrm{CT}$, computed tomography; $\mathrm{Hb}$, hemoglobin; eGFR, estimated glomerular filtration rate; ARB, angiotensin II receptor blocker; ACE, angiotensin converting enzyme; MRA, mineralocorticoid receptor antagonist; CIED, cardiac implantable electronic device 
Table 2. Characteristics of 2 groups after ablation

\begin{tabular}{lccc}
\hline & Group 1 & Group 2 & p value \\
echocardiographic parameters & $\mathrm{n}=9$ & $\mathrm{n}=22$ & \\
follow-up period of echocardiography - $\mathrm{d}$ & $206(181-234)$ & $292(129-449)$ & 0.845 \\
LAD - mm & $41.4(38.7-44.1)$ & $37.4(33.7-42.9)$ & 0.0817 \\
LAV - ml & $62.5(57.5-67.3)$ & $45.2(38.0-51.6)$ & 0.0016 \\
LAVI - ml/m & $34.0(32.6-42.8)$ & $23.7(20.9-27.3)$ & 0.000554 \\
LVEF - \% & $41.1(35.7-54.1)$ & $64.1(61.8-66.5)$ & 0.000018 \\
LV end-diastolic diameter - mm & $49.8(48.5-58.7)$ & $46.9(43.1-49.9)$ & 0.00845 \\
LV end-systolic diameter - mm & $37.7(35.5-50.5)$ & $31.2(28.1-33.7)$ & 0.00118 \\
Moderate or severe MR & $2(22.2)$ & $1(4.5)$ & 0.195 \\
NT-proBNP - pg/ml & $337(72-1242)$ & $104(57-134)$ & 0.344 \\
& & & \\
Recurrence of AF & $2(22.2)$ & $7(31.8)$ & 0.689 \\
Time from ablation to recurrence - $\mathrm{d}$ & 635 & $176(105-397)$ & 0.667 \\
\hline
\end{tabular}

Abbreviations: LAD, left atrial diameter; LAV, left atrial volume; LAVI, left atrial volume index $L V E F$, left ventricular ejection fraction; LV, left ventricular; $M R$, mitral regurgitation; $A F$, atrial fibrillation

(a) LAV-CT

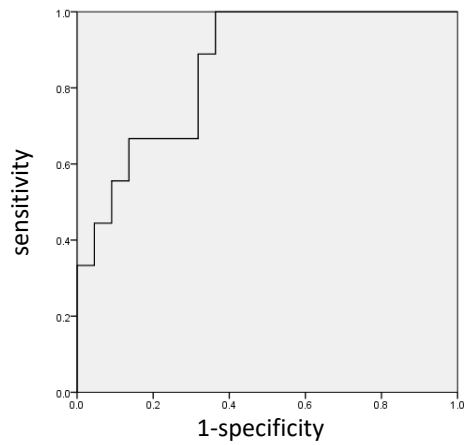

(c) LAVI-echo

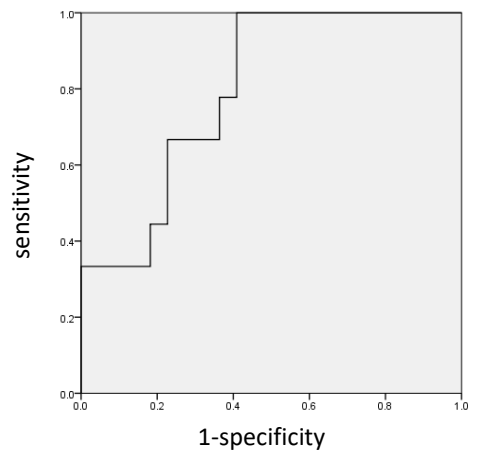

(b) LAVI-CT

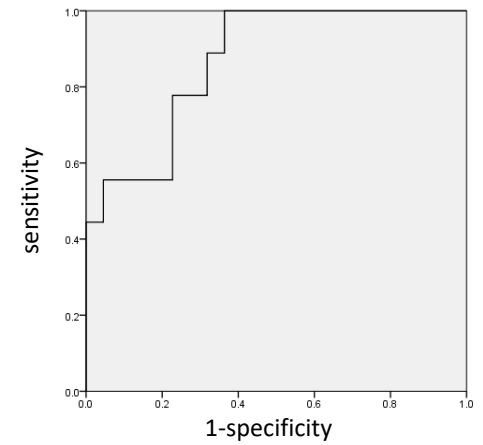

(d) LAD

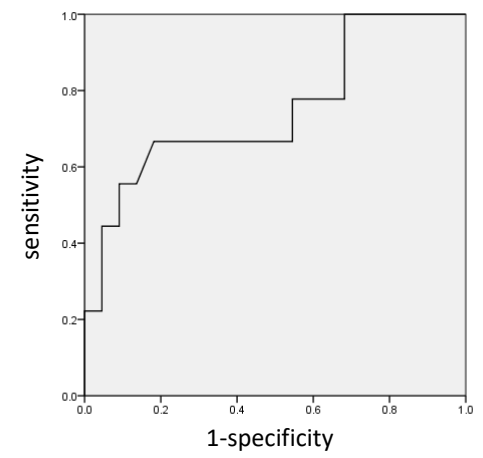

